\title{
Role of resveratrol in cardiovascular and associated diseases.
}

\author{
Shehnai Basharat, Arooj Saeed*,Wajeeha Bashir Baig \\ *Institute of Diet and Nutrition Sciences, University of Lahore, Lahore, Pakistan
}

\begin{abstract}
Resveratrol is a potential bioactive compound found in fruits and taken as dietary supplements. Due to possessing multiple beneficial properties;anti-oxidant, anti-atherosclerosis, cardioprotective, anti-cholesterolemia and glucose regulation. It combats with globally high mortality and morbidity rate diseases. Resveratrol deals with cardiovascular disease and other associated diseases after the result of ROS accumulation. It seems successful in neutraceutical and pharmaceutical to prevent and treat cancer proliferation, diabetes, diabetic-cardiomyocyte, neuropathy, nephropathy and obesity.
\end{abstract}

Keywords: Resveratrol, Diabetes Mellitus, Oxidative stress, cancer, CVD.

Accepted on September 15, 2020

\begin{abstract}
Abbreviations
AMP: Activated Protein Kinase; ATP: Adenosine Triphosphate; CVD: Cardiovascular Disease; CRP: C-Reactive Protein; FoxOs: Forkhead Box Protein 01; HDL: High Density Lipoprotein; LDL: Low Density Lipoprotein; Nrf2: Nuclear Related Factor 2; PGC: Plant Growth Chamber; p-Eno: Phosphorylation of Endothelial Nitric Oxide Synthase; PKB: Phosphoprotein Kinase B; PLG-NP: Polylactic Glycolic Acid Nano Particles; ROS: Reactive Oxygen Species; SIRT 1: Sirtuin-1; SOD: Superoxide Dismutase; T2DM: Type 2 Diabetes Mellitus; TG: Triglyceride.
\end{abstract}

\section{Introduction}

Resveratrol belongs to polyphenol and non-flavonoid structure is present in grapes, barriers, peanuts and red wine. Resveratrol is a natural stilbene and a phytoestrogen that exhibit anti-oxidant, anti-inflammatory, cardio-protective and anti-cancer properties [1]. Due to its different bio-activities, it is approved to take as dietary supplementation. It is considered as efficiently beneficial to many disease handling. Resveratrol is absorbed precisely orally administration and completely absorbed in the intestine [2]. Red wine contains resveratrol with different polyphenols. Many experimental trials have confirmed, phytochemical; resveratrol protects against in heart failure, cancer and other associated diseases like diabetes-cardiac, cardio-oncology and obesity caused by oxidative stress or secondary disease function [3] as they have become overwhelmed the global health [4]. A short term disclosure of resveratrol with high dosages shows extraordinary cardioprotective results [5]. Furthermore, resveratrol is good for kidneys. Resveratrol plays a positive role on cardiovascular diseases and lower down atherosclerosis as well.

Cancer, along with diabetes and CVD, is becoming a disease taking high mortality and morbidity across the world. There are restrictions in present chemotherapies due to occurrence of resistance against the therapy. Many studies have shown resveratrol as influential chemosensitizer agent [6]. It belongs to system of biopharmaceutical classification, as it has less water solubility and high-rise membrane permeability. Resveratrol is phytoalexin polyphenol; produced in plants under stress conditions [7]. Resveratrol encapsulated with casein claimed remarkable oral availability of resveratrol. After oral intake, intestinal absorption can be enhanced by glycosylated PLGA NP of resveratrol. This compound acts as chemo-preventive agent and increases bioavailability $330 \%$. Resveratrol blocks carcinogenesis at initial stages even with low dose concentration. It can change the gene expression that can rule cancer initiation and proliferatio [8]. Therapies against cancer patients may cause toxicity in cardiovascular system. Resveratrol manages the barrier that comes in cancer treatment, which is the drug resistance. The ideal dose for resveratrol supplementation, however, may be less but metabolic disturbance is a risk factor for multiple cancer types and reversal of metabolic disturbances by lowdose resveratrol may reduce cancer risk as well as improve cancer outcomes [9]. The polyphenol resveratrol has antioxidant property. The scientific literature indicates that there is a link between obesity, Diabetes Mellitus, Oxidative stress and cataract formation. Diabetes associated with CVD is more complicated [10]. Long time hyperglycemia leads to overproduction of reaction oxygen species in mitochondria. Resveratrol lower the production of mitochondrial superoxide instead energizes biogenesis in mitochondria. Excessive oxidative stress may cause large scale oxidative damage to proteins, DNA and lipids resulting in damaged cardiomyocyte functions including contractility, ion transport and calcium cycling that results as heart failure, fibrosis, cardiac hypertrophy. Meanwhile, oxidative stress dysfunctions NO;a vasoprotectant to endothelium. This compound increases the expression of various antioxidant enzymes. It has been shown that resveratrol has less direct scavenging activity rather it possesses gene regulatory property. Resveratrol has ability to scavenge $\mathrm{O} 2$ specially produced by xanthine [11]. 


\section{Mechanism of action against oxidative stress}

Resveratrol disassociates Nrf2 from the cytoplasm and bind it where the transmission starts in the nucleus. Resveratrol also activates AMPK; AMP-activated protein kinase, that in terms promote SIRT and FoxOs; forkhead box protein 01. AMPK stabilizes FoxOs structure. They produce antioxidant genes after transcription of FoxOs in the nucleus. SIRT; sirtuin1, also deacetylated PGC-1gama which facilitates in antioxidant gene expression and reduce oxidative stress [12] (Figure 1).

\section{Review of literature}

A recent study evidence showed that resveratrol improves cardiac-oxidative stress and myocardial dysfunctions in diabetes. In this experimental study diabetes induced rats were given resveratrol supplements. The given dose was $50 \mathrm{mg} / \mathrm{kg} /$ day, orally. And the duration of this treatment last 16 weeks. As a result of that trial resveratrol faded diabetes-induced cardiac dysfunction and reduced hypertrophy was observed by increasing EF\% ejection fraction, fraction shortening [13].

In this study, role of C-reactive protein (CRP) has been seen in randomized controlled meta-analysis studies. There was an eminent decrease in CRP with type 2 diabetes, took supplementation of resveratrol. As C-reactive protein (CPR), produced by liver cells, is associated with inflammatory marker. It showed powerful preventive results on CRP in T2DM patients. It decreases the CRP level in people who were taking resveratrol supplements [14].

In another experimental study, resveratrol improved metabolic and neurological system. It was a double blind, placebo controlled interventional study, in which 36 T2DM older (above 49 years) were participated. Dose was given $0,75,150$, $300 \mathrm{mg}$ after interval of one week. Duration of this study was 6 months. The study outcome suggested maximum improvement was observed with the lowest dose used and can enhance basal cerebral blood flow and associated improved learning 9 [15].
The present study is pilot study in which analysis was performed on older participants. As older adults are more prone to CVD. Participants were randomly divided into two treatment groups. One treatment group had 10 participants $(n=10)$ and $300 \mathrm{mg}$ dose of resveratrol was given for 90 days. In second treatment group 1000mg dose was given to 9 participants $(n=9)$. The trial was to study CVD risk biomarker i.e. oxidized low-density lipoprotein. As a result, the study showed higher resveratrol dose of $1000 \mathrm{mg}$, increased CVD risk biomarkers i.e. oxidized low-density lipoprotein, soluble E-selectin-1 and soluble intracellular adhesion molecule-1 in older adults as compared to lower dose treatment group [16] (Table 1).

In a recent clinical study, rats were induced type 1 diabetes that resulted oxidative stress. Rats were grouped in 4 experimental groups with each 8-9 sample size. A was controlled group, B was diabetic group, $\mathrm{C}$ was resveratrol $10 \mathrm{mg}$ treatment group, D was $20 \mathrm{mg}$ resveratrol treatment group. The trial consumed 4 weeks. In this trial, oxidative stress marker (activity of superoxide dismutase, catalase and glutathione peroxide, sulfhydryl group, total oxidant status, soluble proteins) were assessed. The results claimed that the resveratrol supplementation administration showed antioxidant properties after T2DM [17].

In another study, resveratrol evident its eminent chemoprevention property by inhibition of multistage cancer development and its proliferation. But to attain its maximum therapeutic activity, nano-formation is used. As it increases its bioavailability and aqueous solubility. Resveratrol potential outcomes in intracellular signal transduction in carcinogenesis [18].

Regarding to this pre-clinical data of experimental studies, dietary intake of resveratrol like other phytochemicals like curcumin and lycopene, are involved in immune system efficiency, apoptosis in transformed cells, make aware malignant cells to cancer killer cytotoxic T cells [19].

This experimental study in animal models, disclosed unique antioxidant potent quality of resveratrol against diabetic

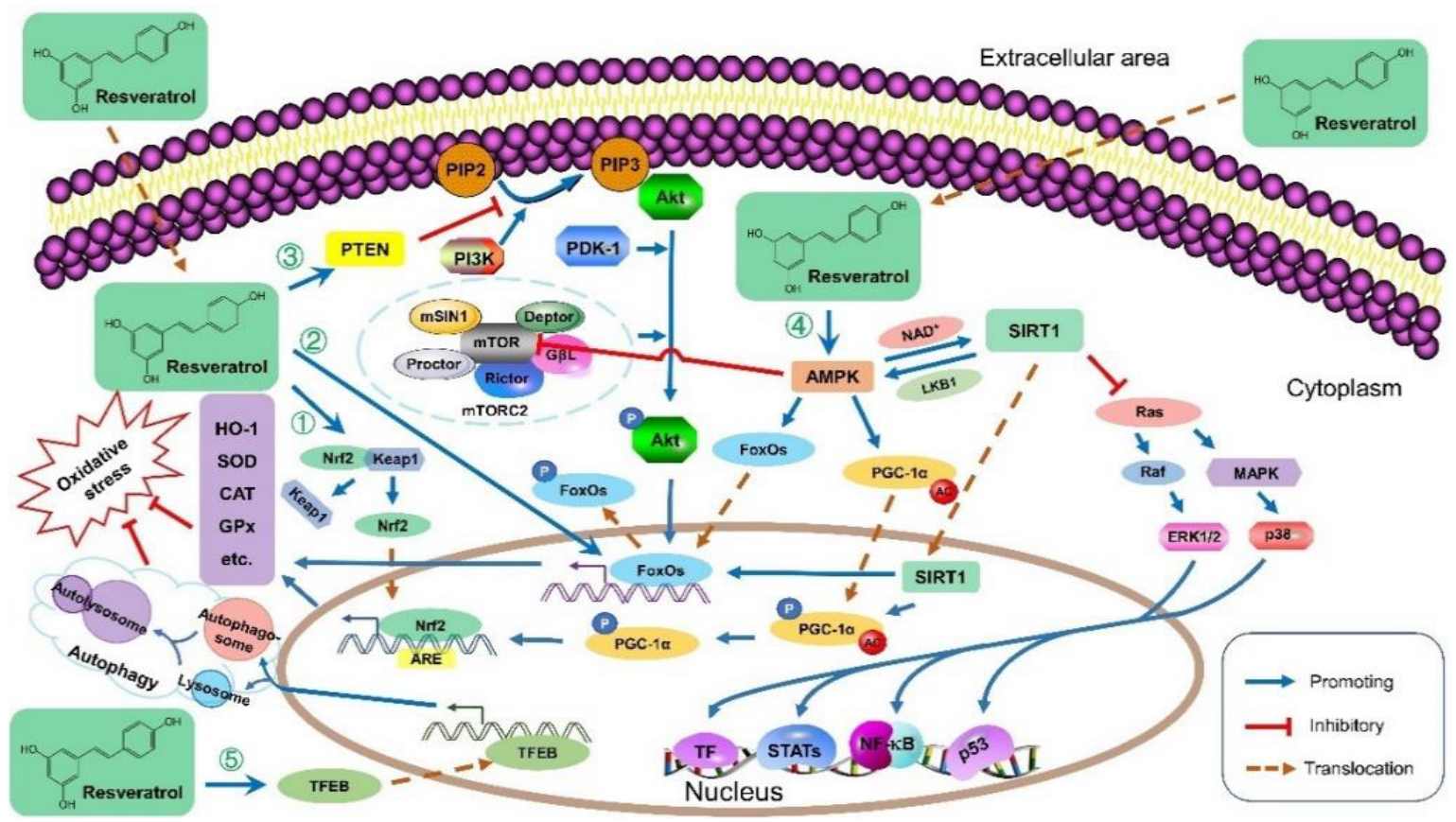

Figure 1. Mechanism of action against oxidative stress. 
Table 1. Original evidences regarding the effect of resveratrol.

\begin{tabular}{|c|c|c|c|c|c|}
\hline Sr.no & Subjects & Dosage & Duration & Outcome & References \\
\hline 1. & 20 Rats & $50 \mathrm{mg} / \mathrm{kg} /$ day, orally & 16 weeks & $\begin{array}{l}\text { Improves cardiac-oxidative stress \& myocardial } \\
\text { dysfunctions in diabetes, and reduced hypertrophy }\end{array}$ & 13 \\
\hline 2. & $\begin{array}{l}36 \text { T2DM older (above } \\
49 \text { years) }\end{array}$ & $\begin{array}{l}0,75,150,300 \mathrm{mg} \text { after } \\
\text { interval of one week. }\end{array}$ & 6 months & $\begin{array}{l}\text { Improved metabolic \& neurological system, lowest } \\
\text { dose of resveratrol can enhance basal cerebral blood } \\
\text { flow \& associated improved learning }\end{array}$ & 15 \\
\hline 3. & $\begin{array}{l}\text { Group } 1(n=10) \\
\text { Group } 2(n=9), \text { older } \\
\text { participants }\end{array}$ & $\begin{array}{l}\text { Group } 1(300 \mathrm{mg}) \\
\text { Group } 2 \text { (1000mg) }\end{array}$ & 90 days & $\begin{array}{c}\text { Higher resveratrol dose increased CVD risk } \\
\text { biomarkers i.e. oxidized low-density lipoprotein, } \\
\text { soluble E-selectin-1 \& soluble intracellular adhesion } \\
\text { molecule-1 in older adults. }\end{array}$ & 16 \\
\hline 4. & $\begin{array}{l}\text { 8-9 in each group, A } \\
\text { was controlled group, } \\
\text { B was diabetic group, } \\
\text { C \& D was resveratrol } \\
\text { treatment group with } \\
\text { different doses. }\end{array}$ & $\begin{array}{c}\text { A was controlled group. } \\
\text { B was diabetic group. } \\
\text { C was } 10 \mathrm{mg} \text { resveratrol, } \\
\& \\
\text { D was } 20 \mathrm{mg} \text { resveratrol } \\
\text { treatment group. }\end{array}$ & 4 weeks & $\begin{array}{l}\text { Resveratrol supplementation administration showed } \\
\text { antioxidant properties after T2DM. }\end{array}$ & {$[17]$} \\
\hline
\end{tabular}

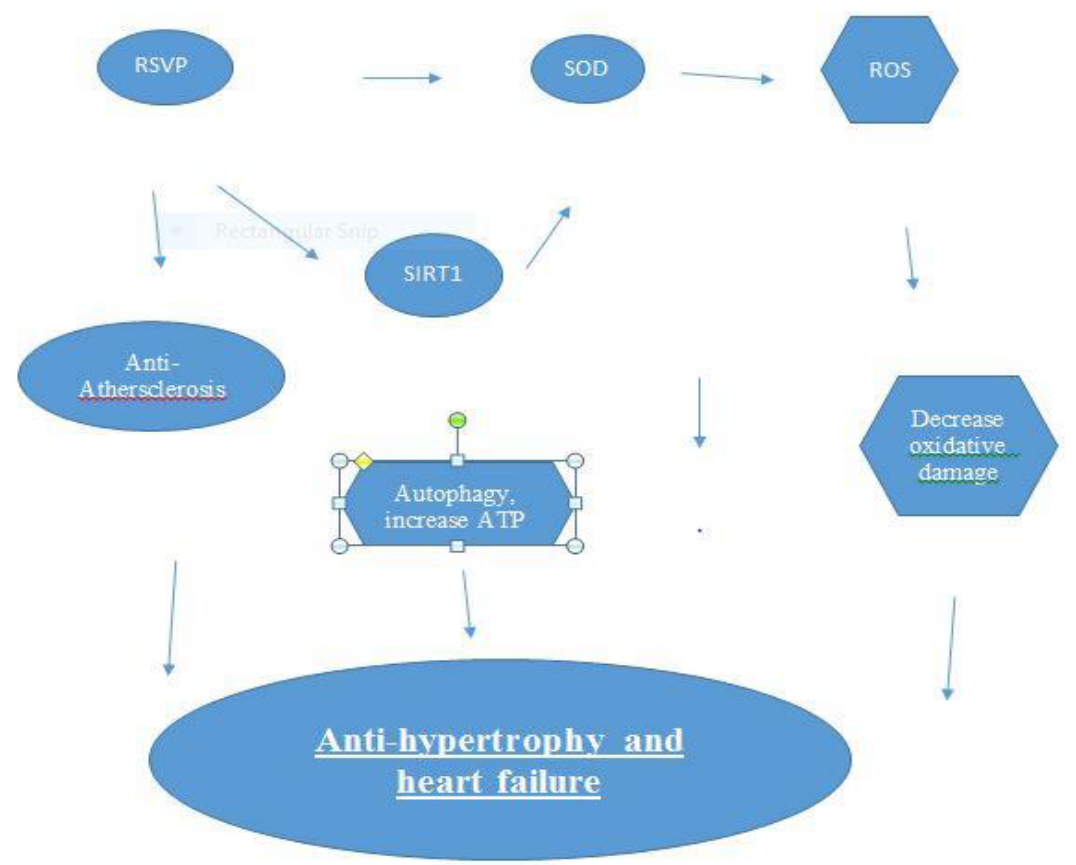

Figure 2. Effect of resveratrol on anti-hypertensive Cardiovascular patient.

cardiomyopathy. Chronic hyperglycemic condition mediates cardiac oxidative stress that lead to cardiac hypertrophy, fibrosis and apoptosis. Oral intake of resveratrol, for four months, enhanced the antioxidant superoxide dismutase enzyme by making left ventricular pressure better, in streptozotocinnicotinamide experimental typr-2 diabetic rats [20].

Furthermore, diabetes also reflects many metabolic malformations. From the studies of different animal and human clinical trials, resveratrol depicted positive outcome against blood glucose level, insulin resistance, pancreatic beta-cells protection and secretion of insulin [21].

Resveratrol has powerful anti-Atherosclerosis properties. In this meta-study, several trials resulted that resveratrol participates against plaque formation by lowering the TG level, LDL and increasing the HDL level into the arteries. Atherosclerosis, is a risk factor for CVDs. In rat studies, its anti-hypercholesterolemia was administered by its pravastatin effect, in the rats fed with high cholesterol diet.

Resveratrol is not fruitful to treat CVD patients in single direction but importantly, it leaves its benefactor cardiovascular impact in diabetic patients. It was studied that in diabetic cardiomyopathy increased oxidative stress, increased apoptosis, diastolic dysfunction, cardiomyocyte hypertrophy happens as secondary function. Basically, resveratrol improved insulin signaling in rat models with rise in SOD, p-eNOS, p-AKT. It has been seen in clinical trials that resveratrol is dose dependent phytochemical. The anti-hypertensive effect of resveratrol (10-320mg/kg/day) has been demonstrated in several animal models. Lower dose did not affect B.P [22] (Figure 2).

In this study, different experimental and clinical trials were studied to know the functions of phytochemicals. Quercitin, Curcumin including resveratrol expressed beneficial results by reducing oxidative stress, lipogenesis and adipogenesis, and chronic inflammation. 
In more, analysis of experimental clinical studies revealed metabolic disorder; obesity control by resveratrol dietary supplementation. Resveratrol controls sirtuin-1 caloric restriction activity and lipid, glucose regulation [23].

\section{Conclusion}

It can be concluded from the previous analysis and meta-analysis studies that higher level of oxidants in the cell, let move to occurance of primay causes of disease and secondary diseases. It was seen this important phytochemical, non-flavonoid, resveratrol plays a novel role in the treatment and prevention of diseases like cancer diabetes mellitus, cardiohypotrphy, CVD and its associated diseases. Resveratrol helps to stop the transcription of gene that produce, oxidant producing enzyme in the cell and that is why resveratrol has taken attention to get from food and dietary supplement as well. It gives beneficial results to major rising health problems.

\section{References}

1. Ko JH, Sethi G, Um JY, et al. The role of resveratrol in cancer therapy. Int J Mol Sci. 2017;18(12): 2589.

2. Elshaer M, Chen Y, Wang XJ, et al. Resveratrol:An overview of its anti-cancer mechanisms. Life Sci. 2018;207: 340-9

3. Zordoky BN, Robertson IM, Dyck JR. Preclinical and clinical evidence for the role of resveratrol in the treatment of cardiovascular diseases. Mol Basis Dis. 2015:1852(6): 1155-77.

4. Abdelgawad IY, Grant MK, Zordoky BN. Leveraging the cardio-protective and anticancer properties of resveratrol in cardio-oncology. Nutrients. 2019:11(3): 627.

5. Ahmet I, Tae HJ, Lakatta EG, et al. Long-term low dose dietary resveratrol supplement reduces cardiovascular structural and functional deterioration in chronic heart failure in rats. Canadian J phy pharmacol. 2017;95(3): 26874.

6. Mohammed S, Harikumar KB. Role of Resveratrol in Chemosensitization of Cancer. InRole of Nutraceuticals in Cancer Chemosensitization. Academic Press. 2018; 61-76.

7. Siddiqui IA, Sanna V, Ahmad N, et al. Resveratrol nanoformulation for cancer prevention and therapy. Ann NY Acad Sci. 2015;1348(1): 20-31.

8. Tabrez S, Jabir NR, Adhami VM, et al. Nanoencapsulated dietary polyphenols for cancer prevention and treatment: successes and challenges. Nanomedicine. 2020;15(11): 1147-62.

9. Guthrie AR, Chow HH, Martinez JA. Effects of resveratrol on drug-and carcinogen-metabolizing enzymes, implications for cancer prevention. Pharmacol Res Persp. 2017:5(1): e00294.

10. Yan F, Sun X, Xu C. Protective effects of resveratrol improves cardiovascular function in rats with diabetes. Expt Therapeu Med. 2018;15(2): 1728-34

11. Xia N, Daiber A, Förstermann U, Li H. Antioxidant effects of resveratrol in the cardiovascular system. British J Pharmacol. 2017;174(12): 1633-46.
12. Meng X, Zhou J, Zhao CN, et al. Health Benefits and Molecular Mechanisms of Resveratrol: A Narrative Review. Foods. 2020;9(3): 340.

13. Fang WJ, Wang CJ, He Y, et al. Resveratrol alleviates diabetic cardiomyopathy in rats by improving mitochondrial function through PGC-1 $\alpha$ deacetylation. Acta Pharmacol Sinica. 2018;39(1): 59-73.

14. Hosseini H, Koushki M, Khodabandehloo H, et al. The effect of resveratrol supplementation on $\mathrm{C}$-reactive protein (CRP) in type 2 diabetic patients: Results from a systematic review and meta-analysis of randomized controlled trials. Comp Therapies Med. 2020;8: 102251.

15. Wong RH, Nealon RS, Scholey A, Howe PR. Low dose resveratrol improves cerebrovascular function in type 2 diabetes mellitus. Nut Met Cardio Dis. 2016;26(5): 393-9.

16. Mankowski RT, You L, Buford TW, et al. Higher dose of resveratrol elevated cardiovascular disease risk biomarker levels in overweight older adults-A pilot study. Exp Gerontol. 2020;131: 110821.

17. Portillo MP, Fernandez-Quintela A. Benefits of Resveratrol Supplementation. MDPI. 2019; 23.

18. Siddiqui IA, Sanna V, Ahmad N, et al. Resveratrol nanoformulation for cancer prevention and therapy. Ann New York Academy Sci. 2015;1348(1): 20-31.

19. Kotecha R, Takami A, Espinoza JL. Dietary phytochemicals and cancer chemoprevention: a review of the clinical evidence. Oncotarget. 2016;7(32): 52517.

20. Mohammadshahi M, Haidari F, Soufi FG. Chronic resveratrol administration improves diabetic cardiomyopathy in part by reducing oxidative stress. Cardiol J. 2014;21(1):39-46.

21. Truong J. The Cytotoxic Effects of Methylmercury on Cardiomyocytes: A Possible Implication for Heart Diseases? (Doctoral dissertation, University of Ottawa).

22. Prasad K. Resveratrol, wine, and atherosclerosis. Int J Angiol. 2012;21(01): 7-18.

23. Aguirre L, Fernández-Quintela A, Arias N, et al. Resveratrol: anti-obesity mechanisms of action. Molecules. 2014;19(11): 18632-55.

\section{*Correspondence to:}

Arooj Saeed

Institute of Diet and nutrition sciences

University of Lahore

Pakistan

Tel: 03491050074

E-Mail: aroojsaeed27@gmail.com 